\title{
Der katholische Rundfunk im niederländischen Rundfunksystem von 1925 bis 1975
}

\author{
von Joan Hemels
}

\section{Die Entstebung und die separative Phase des niederländischen Rundfunksystems}

Nichts erweist sich bei der Behandlung der Geschichte der Massenmedien gefährlicher als zu behaupten, jemand habe mit etwas den Anfang gemacht. In einer Reihe von Publikationen erhält der niederländische Ingenieur H. H. S. à Steringa Idzerda, Eigentümer der Nederlandsche Radio Industrie (NRI = Niederländische Rundfunkindustrie), die Ehre, am 6. November 1919 als erster eine durch Anzeige im voraus angekündigte Rundfunksendung für ein möglichst großes und breit gestreutes Publikum durchgeführt $\mathrm{zu}$ haben. Seine Rundfunkstation "PCGG“ in Den Haag bestand bis 1924․ Am 21. Juli 1923 begann die Nederlandsche Seintoestellen Fabriek (NSF = Niederländische Sendegerätefabrik), mit einem Versuchssender auszusenden. Das führte im gleichen Jahr zur Gründung des Comité De Hilversumsche Draadlooze Omroep (HDO $=$ Hilversumer drahtloser Funk). Die NSF begann darauf mit der fabrikmäßigen Herstellung von Empfangsgeräten: die Sendungen schufen den Bedarf. Aus dem HDO entstand nach einer Namensänderung und einer Fusion schließlich am 1. Januar 1928 die Algemeene Vereenigung Radio Omroep (AVRO = Allgemeine Rundfunkvereinigung). Obwohl die AVRO vorgab, ein allgemeiner Rundfunk für Hörer mit unterschiedlichen weltanschaulichen Überzeugungen zu sein, entstand dennoch ein pluralistisches Rundfunksystem, das gekennzeichnet wurde durch das typische niederländische Phänomen der „verzuiling“.

Dieser Begriff „verzuiling“ (Versäulung) bezeichnet, sofern man die Struktur der niederländischen Gesellschaft beschreiben will, jene Erscheinung, die als (weltanschaulich-)pluralistisches Ordnungsprinzip die niederländische Gesellschaft maßgeblich bestimmte bzw. noch bestimmt. Der Aufbau des öffentlichen Lebens nach konfessionellen und weltanschaulichen Gesichtspunkten spiegelte sich bis Mitte der sechziger Jahre dieses Jahrhunderts in der Presse, im Rundfunk, in den Fach- und Berufsorganisationen, im Volksgesundheitswesen, in den Sportvereinigungen, in der Sozialarbeit, in den Jugend- und Kulturbewegungen und im Unterrichtswesen auf allen Ebenen. Dieses Ordnungsprinzip wurde aber im letzten Jahrzehnt in starkem Maße untergraben ${ }^{2}$.

Nacheinander wurden als weltanschauliche Rundfunkgesellschaften gegründet: die Nederlandsche Christelijke Radio Vereeniging (NCRV = Niederländische Christliche Rundfunkvereinigung) $1924^{3}$, der Bond van Roomsch Katholieke Radio Vereenigingen (Verband römisch-katholischer Rundfunkvereinigungen) 1925 - seit 1926 "Stichting Katholieke Radio Omroep" (KRO = Stiftung Katholischer Rundfunk) genannt -, die Vereeniging van Arbeiders Radio Amateurs (VARA = Arbeitervereinigung der Rundfunkamateure) 1925 - heutzutage Omroepvereniging Vara genannt -, und der Vrijzinnig Protestantsche Radio Omroep (VPRO = Freisinnig

Dr. Joan Hemels ist wissenschaftlicher Mitarbeiter am Institut für Massenkommunikation der Katholischen Universität in Nimwegen (Niederlande). 
Protestantischer Rundfunk) 1926, der jetzt Omroepvereniging VPRO heißt. Die VPRO war aus prinzipiellen Gründen gegen eine Aufteilung des Rundfunks nach weltanschaulichen unterschiedlichen "Säulen" und trat für einen nationalen, die Toleranz fördernden Rundfunk ein. Sie weigerte sich jedoch, die AVRO als repräsentative nationale Rundfunkgesellschaft anzusehen und stellte darum ihre eigene Vereinigung für das Aussenden religiöser Sendungen zur Verfügung. Erst nach dem Zweiten Weltkrieg begann die VPRO, auch andere als typisch religiöse Programme und kirchliche Dienste auszusenden. In der Zeit von 1925 bis 1929 unternommene Bestrebungen, einen einzigen nationalen Rundfunk einzurichten, schlugen fehl. Die AVRO wurde mehr oder minder gegen ihren Willen gezwungen, dic Rolle einer fünften, neutralen, aber vorwiegend liberal ausgerichteten „Säule“ zu spielen". Für das Wachstum der genannten und viel später gegründeten Rundfunkgesellschaften verweisen wir auf Tabelle 1 .

Tabelle I: Entwicklung der Mitgliederzahlen der niederländischen Rundfunkgesellschaften in Tausendern.

\begin{tabular}{lrrrrrrrrrrrr} 
& 1930 & 1939 & 1947 & 1950 & 1955 & 1965 & 1970 & 1971 & 1972 & 1973 & 1974 & 1975 \\
\hline AVRO & 180 & 197 & 86 & 285 & 312 & 385 & 807 & 736 & 848 & 830 & 835 & 827 \\
KRO & 80 & 161 & 85 & 275 & 439 & 567 & 516 & 542 & 547 & 544 & 530 & 542 \\
NCRV & 70 & 126 & 101 & 240 & 362 & 465 & 459 & 471 & 479 & 480 & 477 & 488 \\
VARA & 70 & 109 & 101 & 210 & 378 & 485 & 471 & 485 & 488 & 492 & 493 & 493 \\
VPRO & 9 & $?$ & 46 & 120 & 227 & 160 & 112 & 139 & 143 & $?$ & 145 & 144 \\
TROS & & & & & & $60^{*}$ & 255 & 280 & 331 & 393 & 500 & 600 \\
EO & & & & & & & & 30 & 137 & 150 & 145 & 141
\end{tabular}

* Stand: März 1966

Quelle: Die Zahlen für 1930 bis 1965: H. Schaafsmax: Mirror of a pillarized society: broadcasting in the Netherlands, in: "Delta " 9:1966/67, Nr. 4, S. 57-69, hier S. 60, und G. P. Bakker: Vijftig jaar en verder. Geschreven ter gelegenheid van het 50 -jarig bestaan van de VARA op 1 november 1975, Hilversum 1975, S. 10, 24 u. 56; die übrigen Zahlen erhielt der Verfasser, J. Hemels, durch Nachfrage bei den verschiedenen Rundfunkgesellschaften.

Im Jahre 1930 kamen das Rundfunk-Reglement mit Normen für den Erhalt einer Sendeerlaubnis und das erste Sendezeitdekret heraus. Für die weitere Entwicklung des Rundfunksystems waren diese, auf dem Rundfunkgesetz von $1928^{5}$ basierenden Eingriffe seitens der Regierung in Sachen Rundfunkgesellschaften, die aus Privatinitiative entstanden, von besonders großer Bedeutung. Die vier großen Rundfunkgesellschaften (AVRO, KRO, NCRV und VARA) konnten über je ein Viertel der Sendezeit abzüglich fünf Prozent verfügen. Dieser Prozentsatz wurde der VPRO und einigen anderen kleinen Sendeberechtigten wie der Radio-Volksuniversiteit (RundfunkVolksuniversität) und dem Humanistische en Idealistische Radio Omroep (HIRO = Humanistischer und Idealistischer Rundfunk) vorbehalten.

In der Zeit vor dem Zweiten Weltkrieg blieb der Rundfunk eine Angelegenheit der Privatinitiative. Eingriffe seitens der Regierung blieben begrenzt und erfolgten auf staatsrechtlicher Grundlage: entweder mußte die Notwendigkeit der Verteilung der knappen Sendefrequenzen und die Mitverantwortung für ihren angemessenen Gebrauch mit dem Blick auch auf ausländische Interessen erfolgen, oder aber die Regierungssorge für die Sicherheit des Staates, die Offentliche Ordnung und die guten Sitten rechtfertigten die repressive und auch präventive Bemühung um die Rundfunkprogramme (über die Radio-Omroep-Controle-Commissie [Rundfunk-Kontroll-Kommission] 1930-1940). 
Seit 1927 nahm die Zusammenarbeit zwischen den Rundfunkgesellschaften einen ersten Anfang in dem Beratungsorgan Comité van Omroepvereenigingen (Komitee der Rundfunkvereinigungen) (ohne AVRO), das 1936 durch das Centraal Bureau van den Omroep (Rundfunk-Zentralbüro) ersetzt wurde, an dem die AVRO jedoch beteiligt war. Vor 1940 verfügten die Rundfunkgesellschaften nur über Einkünfte aus den Beiträgen der Mitglieder bzw. der Sympathisanten und aus dem Vertrieb der Rundfunkprogrammhefte.

Während des Zweiten Weltkrieges wurde am 12. März 1941 „De Nederlandsche Omroep" (Der Niederländische Rundfunk), ein vollständig gleichgeschalteter nationaler Reichsrundfunk, gegründet. Die Rundfunkgesellschaften, unter ihnen auch die KRO, wurden aufgehoben und ihr Eigentum beschlagnahmt ${ }^{\natural}$.

\section{Die Gründung des Katholieke Radio Omroep (KRO)}

Im September 1924 wurde der Vorstand der Apologetische Vereeniging Petrus Canisius $(\mathrm{AVPC}=$ Apologetische Vereinigung Petrus Canisius) von verschiedenen Seiten darauf aufmerksam gemacht, daß andere Kirchengemeinschaften schon tatkräftig vom Rundfunk Gebrauch machten?. Die AVPC wurde sich bewußt, daß dieses neue Medium auch für die Katholiken eine Rolle in der Verkündigung und Verteidigung des Glaubens spielen könnte. Am 27. Januar 1925 beschloß sie, eine Kommission zusammenzustellen, die das Rundfunkproblem für Katholiken untersuchen sollte, um eine Rundfunkvereinigung zu gründen. Diese sollte dann bei der Regierung eine Sendeerlaubnis beantragen. Sendezeit wäre dann in Hilversum von der NSF käuflich zu erwerben, oder aber man würde eine eigene Sendestation bauen können.

Am gleichen 27. Januar 1925 berichtete die katholische überregionale Tageszeitung „De Tijd“ über Pläne, nach denen man eine katholische Rundfunkvereinigung von Rundfunkamateuren gründen wolle. Am 4. Februar des Jahres wurde in einem Bericht in „De Tijd" und anderen Zeitungen um Sympathiebekundungen für eine ebenfalls zu gründende katholische Rundfunkgesellschaft gebeten, die Sendungen aufnehmen könnte. Die Interessenten wurden gebeten, in Kontakt mit dem Amsterdamer Pfarrer L. H. Perquin O.P. zu treten. Am 6. Februar veröffentlichte „De Tijd" einen Artikel von Pfarrer E. Ribbergh aus St. Gerlach über den Rundfunk und die Möglichkeiten dieses Mediums für die katholische Sache. Ribbergh, der über die Amsterdamer Initiative nicht unterrichtet war, schrieb auch, daß bereits 1924 in der Kammer Bemühungen (durch die AVPC?) unternommen worden seien, um eine katholische Sendestation einzurichten.

Der Dominikaner Perquin (1864-1938) war - vor seiner Ernennung 1919 zum Pfarrer in Amsterdam - ab 1906 Zensor von "De Maasbode“ (Rotterdam) gewesen. In Amsterdam übernahm er auch die Leitung des 1911 als kleines Anzeigenblatt angelaufenen Wochenblatts „De Bazuin", das zunehmend apologetischen Charakter hervorkehrte. Im Rundfunk sah Perquin ein mächtiges Mittel, um die katholische Weltanschauung bekannt zu machen und um das Apostolat zu ergänzen. Überdies sollte der katholische Rundfunk der neutralen Lebensauffassung und dem Gedankengut Andersdenkender, das Katholiken verwirren könnte, entgegenwirken.

Bereits am 24. Februar 1925 wurde das kurz zuvor in Amsterdam gegründete radiocomité (Rundfunk-Komitee) in eine „R[oomsch] K[atholieke] Radiovereeniging “ (Römisch Katholische Rundfunkvereinigung) umgebildet. Derartige Rundfunkvereini- 
gungen von Hörern und Rundfunkamateuren wurden 1925 vielerorts gegründet; in Den Haag wurde eine katholische Rundfunkvereinigung noch früher errichtet als in Amsterdam, nämlich am 5. Februar. Im Frühjahr 1925 wurde bekannt, daß die Bischöfe die Gründung vieler katholischer Rundfunkvereinigungen begrüßten und wünschten, eine katholische Rundfunkgesellschaft möge $\mathrm{zu}$ senden beginnen. Auf Vorschlag des Episkopats kamen am 23. April 1925 die Vorstände der katholischen Rundfunkvereinigungen unter dem Vorsitzenden der AVPC, dem Priester D. W. van Koeverden, der Perquin Unterstützung und Mitarbeit geboten hatte, zusammen. Sie gründeten den überregionalen Nederlandsche Bond van R. K. Radiovereenigingen (Niederländischen Verband der Römisch Katholischen Rundfunkvereinigungen) und stellten ihn unter den geistlichen Schutz des heiligen Willibrord.

Am 1. Mai 1925 erschien die erste Nummer von „De R. K. Radio-gids“ (Der R. K. Rundfunkführer) als offizielles Organ des Nederlandsche Bond van R. K. Radiovereenigingen en van den Katholieken Draadloozen Omroep. Das Blatt, das noch nicht der KRO gehörte, brachte zu einem bedeutenden Teil lediglich technische Informationen für Rundfunkamateure. Am 4. Oktober desselben Jahres fand die erste Sendung statt: mit einer Tageseinführung und einem Hochamt, die beide aus der Pfarrkirche Perquins gesendet und von der HDO unter Leitung des bahnbrechenden Rundfunkmanns Willem Vogt durchgeführt wurden. Am 24. November fand die erste offizielle Sendung der KRO statt. Das abendfüllende Programm wurde von einem bekannten Hochschullehrer der Katholischen Universität Nijmegen, Prof. Dr. G. Brom, eröffnet, der eine Rede hielt über das Thema „Een gebed door de lucht " (Ein Gebet über den Äther).

Im Frühjahr 1926 wurde - wiederum auf Ersuchen des Episkopats - über die juridische Form der KRO mit allen großen katholischen Organisationen, darunter auch dem Bond van R. K. Radiovereenigingen, verhandelt.

Gewählt wurde die Stiftungsform, wobei bemerkt sei, daß die Stiftung keine Mitglieder mit gesetzlich umschriebenen Rechten und Pflichten kennt, sondern nur Sympathisanten oder auch zahlende Mitglieder. (Im Folgenden wird zwischen Mitgliedern und Sympathisanten nicht mehr eigens unterschieden, sondern nur noch von Mitgliedern gesprochen.) Zum Vorsitzenden des ersten Vorstandes der Stichting Katholieke Radio Omroep (Stiftung Katholischer Rundfunk) wurde am 12. April 1926 Perquin gewählt. Außer einem vier Personen zählenden Vorstandsausschuß wurde auch ein Gesamtvorstand gebildet. Nach den Worten Perquins waren darin alle Ränge und Stände vertreten - durch Personen, bei denen man genügend Bildung voraussetzen konnte, um in Sachen Kultur führend zu sein. In Artikel 2 der Statuten wurde als Ziel angegeben, der römisch-katholischen Weltanschauung in ihrer gesamten Vielfalt Ausdruck zu verleihen.

Arţikel 3 zählt als Mittel und Wege dazu auf: Hörfunk-Sendungen zu besorgen und Werbung in der Presse wie andernorts durchzuführen. Später wurde hinzugefügt die Herausgabe eines Rundfunkprogrammhefts als eines offiziellen Organs; in denselben Statuten wurde als Aufgabe der KRO genannt, zu verkündigen und in Sachen von Religion und Kultur Führung zu geben, und dazu als weitere Aufgabe, hochstehende Unterhaltung zu vermitteln.

Die Vorstände und besonders die Vorsitzenden der KRO und die protestantischchristliche NCRV arbeiteten hin und wieder bewußt zusammen. Sie bauten gemeinsam einen eigenen Sender (Huizen, 1927) und nahmen als christlich orientierte 
Rundfunkgesellschaften wiederholt denselben Standpunkt in rundfunkpolitischen Fragen ein. Nach Aussage des Archivars der KRO, Heinz Joosten, schlug der Vorsitzende der NCRV, A. van der Deure, Perquin 1928 mit Erfolg für eine königliche Auszeichnung vor. Die KRO gehörte 1927 zu den ersten Mitgliedern des Bureau Catholique International de Radiodiffusion (Internationales Katholisches Rundfunkbüro) (heute Unda: Association Catholique Internationale pour la Radiodiffusion et la Télévision [Internationale Katholische Vereinigung für Rundfunk und Fernsehen]). Die KRO war auch von Anfang an mitbeteiligt an der 1925 in Genf gegründeten Union Internationale de Radiophonie (Internationale Rundfunkunion) (heute: Union Européenne de Radiodiffusion et Télévision [Europäische Rundfunkund Fernsehunion] $)^{7}$.

Die Studios der KRO waren anfänglich in Amsterdam und später in Hilversum angesiedelt. Im Jahre 1933 begann die KRO mit dem Einsatz des Kurzwellendienstes Philips Omroep Holland-Indie (PHOHI = Philips-Funk Holland-Indien), der für Missionare in Ost- und Westindien sendete. Seine Programme für eine spezifische Gruppe in den Kolonien entwickelten sich zu Weltsendungen für alle Niederländer in der Fremde. Man kann diese Sendetätigkeiten als eine Art Vorgeschichte für die am 15. April 1947 errichtete Stichting Radio Nederland Wereldomroep (Stiftung Niederländischer Weltrundfunk) ansehen.

\section{Der Kampf um die Wiederberstellung der Rechte und die konföderative Phase des Rundfunks nach 1947}

Nach der Befreiung der Niederlande von der deutschen Besatzung Anfang Mai 1945 wurden die Rundfunksendungen anfänglich durch Radio Herrijzend Nederland (Rundfunk der wiedererstehenden Niederlande) besorgt, ein Organ der Militair Gezag (Militärbehörde), die die Macht auf Zeit bis zur Ubernahme durch eine zivile Verwaltung übernommen hatte. Die Rundfunkaufgabe wurde am 19. Januar 1946 von der am 27. Juli 1945 gegründeten Stichting Radio Nederland in Overgangstijd (Stiftung Rundfunk der Niederlande in Übergangszeit) übernommen. In dieser Stiftung arbeiteten die Rundfunkgesellschaften bis zur Wiederherstellung ihrer Rechte zusammen. Noch während des Krieges, nämlich am 15. Mai 1944, hatten sie, mit Ausnahme der VPRO, in der Federatie van Omroepvereenigingen (Föderation der Rundfunkvereinigungen) ein Organ zur Wahrnehmung gemeinsamer Interessen geschaffen. Nach zahlreichen politischen Verwicklungen und u.a. dem mißglückten Versuch, einen nationalen Rundfunk nach dem Vorbild des britischen BBC zu gründen, wurden am 1. März 1947 dieselben Sendeberechtigten, wie sie bereits vor dem Krieg bestanden hatten, wieder eingesetzt. Anfang 1947 hatten sie unter Druck der Regierung gemeinsam die Nederlandse Radio Unie (NRU $=$ Niederländische Rundfunkunion) gegründet, um künftig zusammen die technischen, künstlerischen und administrativen Belange wahrnehmen zu können. Überdies wurde die NRU beauftragt, ein gemeinsames (nicht: allgemeines) Programm zu besorgen, das ungefähr acht Prozent der gesamten Sendezeit ausmachte. Die Kirchengemeinschaften bekamen auch Sendezeiten zugewiesen; sendeberechtigt wurden: die Rooms Katholiek Kerkgenootschap (RKK = Römisch-Katholische Kirchengemeinschaft), der Convent van Kerken inzake Radiodiensten ( $\mathrm{CvK}=$ Kirchenkonvent für Rundfunkdienste) und das Interkerkelijk Overleg in Radioaangelegenheden (IKOR $=$ Interkirchlicher Rat in Rundfunkangelegenheiten), wobei $\mathrm{CvK}$ und IKOR gemeinsame Arbeitsorgane unterschiedlicher nicht-katholischer Kirchengemeinschaften sind. 
Die zunehmende Zusammenarbeit zwischen den Rundfunkgesellschaften nach 1947 führte dazu, daß die Zeit bis 1965 im Gegensatz zur separativen Phase der Vorkriegszeit als konföderative Phase bezeichnet wird. Namens des für den Rundfunk verantwortlichen Ministers wurde ein Regierungskommissar zur Aufsicht über die Sendeberechtigten bestellt. Die Finanzierung des Rundfunks wurde im Gegensatz zu der Zeit vor 1940 teilweise Regierungsangelegenheit. Die vor dem Krieg bereits vorbereitete, aber erst durch die deutsche Besatzung eingeführte Hörfunkgebühr blieb bestehen. Die N. V. Nederlandsche Omroep Zender Maatschappij (N. V. Nozema = Niederländische Rundfunksender-Gesellschaft $A G$ ), errichtet aufgrund des Rundfunk-Sendergesetzes vom 22. Juli 1935, blieb verantwortlich für den Bau, die Verwaltung und den Betrieb der Sendeeinrichtungen. Die Anteile der N. V. Nozema blieben in Händen der PTT (Staatsbetrieb für Post, Telegraphie und Telephon) (60 Prozent) und der vier großen Rundfunkgesellschaften aus der Vorkriegszeit (AVRO, VARA, KRO und NCRV je 10 Prozent). Über die PTT blieb der Minister für Verkehr für den Senderpark verantwortlich.

Nachdem die N. V. Philips zwei Jahre hindurch mit dem Fernsehen experimentiert hatte, begann am 2. Oktober 1951 in den Niederlanden die Zeit der offiziellen Sendungen des neuen Mediums. Analog der NRU wurde die "Nederlandse Televisie Stichting" (NTS = Niederländische Fernsehstiftung) ins Leben gerufen. Die NTS hatte für die technische Einrichtung zu sorgen, bekam aber (noch) keine ProgrammAufgabe. Die Rundfunkgesellschaften besorgten reihum unter eigenem Namen die Programme. Der Regierungskommissar für den Rundfunk wurde namens der Regierung auch mit der Aufsicht über das Fernsehen beauftragt. Im Jahre 1954 übernahm die Regierung die Finanzierung der Fernsehsendungen von den Rundfunkgesellschaften; seit 1955 wird eine Fernsehgebühr erhoben. Aufgrund des Fernsehdekrets von 1956 konnte der für Rundfunkangelegenheiten verantwortliche Minister drei sogenannte Kronmitglieder für die Verwaltung der NTS ernennen und so den Regierungseinfluß verstärken. Von der insgesamt für Fernsehsendungen verfügbaren Zeit mußten mindestens 25 und durften höchstens 50 Prozent "gemeinsam" und unter der Verantwortung der NTS produziert und gesendet werden. In der Mitte der fünfziger Jahre begannen auch die Auseinandersetzungen über kommerzielles Fernsehen und wurden Initiativen für die Ausstrahlung von Werbesendungen entwickelt.

Im Herbst 1959 wurde der Vrije Radio Omroep Nederland (VRON = Freie Rundfunk Niederlande) gegründet; am 25. April 1960 sendete dann "Radio Veronica“ von einem in der Nordsee unter Panamesischer Flagge fahrenden Senderschiff aus das erste Hörfunkprogramm. Die Zeit der "Ätherpiraten“, die vor der Küste von Schiffen aus operierten, ging für die Niederlande erst am 31. August 1974 zu Ende. Im Oktober 1965 begann der dritte Hörfunksender, Hilversum III, als „Pop-Stationim-Werden" mit "Veronica“ zu konkurrieren. Im Jahre 1964 erlebten die Niederlande zur großen Úberraschung der ausländischen Medien kurzfristig Hör- und Sehfunksendungen eines zweiten Piratensenders, der Reclame Exploitatie Maatschappij (REM $=$ Werbenutzungsgesellschaft), von einer eigens erbauten treibenden "Insel ${ }^{*}$ vor der Küste ${ }^{8}$.

Das legale zweite Fernsehnetz wurde am 1. Oktober 1964 offiziell in Betrieb genommen. Sowohl für die Geschichte der niederländischen parlamentarischen Demokratie als auch für die Geschichte des niederländischen Rundfunks bleibt die am 26. Februar 1965 zutagegetretene Kabinettskrise ein bemerkenswertes Ereignis. Das Kabinett scheiterte an der Frage, wie das Rundfunksystem endlich definitiv geregelt 
werden sollte. In der Mitte der sechsziger Jahre standen die alten Rundfunkgesellschaften unter schwerem Druck. Dafür gab es zwei Gründe: einerseits war seit 1960 der Prozeß der „Entsäulung“ in Gang gekommen, andererseits strebten mehrere Interessengruppen den kommerziellen Rundfunk anstelle oder neben dem „versäulten" Rundfunksystem an. In der Innenpolitik erwies sich die Rundfunkproblematik als sehr heikel, so daß das Kabinett sich von Anfang an vielen Fußangeln und Engpässen gegenübersah. Die römisch-rote Koalition zerfiel wegen fehlender Einmütigkeit über folgende Fragen: ob neue Sendeberechtigte unter der Losung "Wie offen ist unser Rundfunksystem?" zugelassen oder nicht zugelassen werden sollten, wie die Sendeberechtigten zu noch mehr Zusammenarbeit bewogen werden könnten und ob Werbung im Hör- und Sehfunk zugelassen werden sollte oder nicht. Die politischen Parteien fanden eine Lösung der vielen Fragen und Probleme, als sie nach intensiven Verhandlungen zu Absprachen über den Rundfunk gelangten. Diese machen zusammen das Rundfunk-Abkommen vom 30. März 1965 aus. Die neue Regierung brachte noch gerade vor Ende 1965 eine vorläufige gesetzliche Regelung für den Rundfunk zustande; diese bestand aus dem Rundfunkdekret 1965, dem geänderten Fernsehdekret 1956 und dem Dekret über Einrichtung eines Werberats. Die Neuregelungen traten am 1. Dezember 1965 in Kraft und sollten bis zu dem Zeitpunkt gültig bleiben, zu dem ein ganz neues Rundfunkgesetz formuliert, angenommen und in Kraft getreten sein würde. Da diese Voraussetzungen erst am 29. Mai 1969 erfüllt waren, nennt man die genannte Zeit die Zeit des „Übergangssystems“.

\section{Das Merkmal der Offenheit im Rundfunksystem seit 1965}

Die Grundlagen des heutigen niederländischen Rundfunksystems wurden in den zwanziger Jahren gelegt. Nach dem Zweiten Weltkrieg erfuhr dieses System keine grundlegenden Veränderungen, sofern nicht das Einsetzen des Fernsehens indirekt die Struktur des Rundfunks beeinflußt hat. Die Zusammenarbeit auf technischem Gebiet und das Regierungsbemühen nahmen merklich zu, aber vor dem 1. Dezember 1965 gab es für neue Sendeberechtigte keine Möglichkeit. Während der zu diesem Zeitpunkt angelaufenen Übergangsperiode eilte man den Regelungen des späteren Rundfunkgesetzes bereits voraus. Die erste neue Rundfunkgesellschaft, die von der Offenheit des Systems profitieren konnte, war die Televisie en Radio Omroep Stichting (TROS $=$ Fernseh- und Rundfunkstiftung), entstanden aus dem REMVersuch (1964). Am 11. Mai 1966 bekam die TROS Sendeerlaubnis und das Recht, ab 1. Oktober desselben Jahres als Rundfunkgesellschaft mit Bewerberstatus ihre Sendungen zu beginnen.

Eine zweite neue Rundfunkgesellschaft war der Evangelische Omroep (EO = Evangelischer Rundfunk), der am 1. April 1970 als Rundfunkgesellschaft mit Bewerberstatus Sendeerlaubnis erhielt. War die TROS gegründet worden, um möglichst viele Unterhaltungsprogramme anzubieten, so wurde die EO von beunruhigten und konservativen Protestanten errichtet, die die NCRV zu progressiv fanden. Im Gegensatz zur TROS bekam die EO Sendeerlaubnis, nachdem die Rundfunkfrage eine definitive gesetzliche Regelung gefunden hatte. Das Wet van 1 maart 1967 tot regeling van de omroep (Gesetz vom 1. März 1967 zur Regelung des Rundfunks) - wir nennen es kurz Rundfunkgesetz - wurde nämlich am 29. Mai 1969 und das Wet op de omroepbijdragen (Rundfunkgebührengesetz) am 1. Januar 1969 rechtskräftig日. Die Grundmerkmale des Rundfunkgesetzes sind die Öffnung für das Hinzutreten neuer 
Sendeberechtigter (die sogenannte „Offenheit" des Rundfunksystems) und die Verpflichtung zur Zusammenarbeit. Das Rundfunkgesetz ist ein Kompromiß, weil es Raum läßt für die traditionellen weltanschaulichen Rundfunkgesellschaften und zugleich die Zulassung neuer Rundfunkgesellschaften vorsieht. Einrichtungen, die über genügend Mitglieder verfügen und beweisen können, daß sie im Publikum lebende kulturelle, religiöse und geistige Bedürfnisse erfüllen und darum von allgemeinem Nutzen sind, können für die Zuteilung von Sendezeit in Betracht kommen $^{10}$. Sie dürfen u. a. keinen Gewinn anstreben und müssen ihre Hauptaufgabe im Besorgen von Hör- und Sehfunksendungen sehen. Rundfunkgesellschaften, die mindestens 100.000 eingeschriebene Mitglieder haben, die zugleich ihre Gebühren an die Rundfunkgebührenstelle des PTT entrichtet haben, können als Rundfunkgesellschaft der Kategorie C Sendezeit erhalten; steigt die Anzahl der Mitglieder über 250.000, dann kann die betreffende Rundfunkgesellschaft zur Kategorie B zugelassen werden, und schließlich besteht bei einer Mitgliedschaft von mindestens 400.000 Personen die Möglichkeit, in die Kategorie A aufzurücken. Gesellschaften, die die allgemeinen Voraussetzungen des Rundfunkgesetzes erfüllen, aber noch keine 100.000, jedoch mindestens 40.000 Mitglieder haben, können den Bewerberstatus einer Rundfunkgesellschaft erhalten, mit einer begrenzten Anzahl Sendestunden ihre Sendungen aufnehmen und innerhalb von höchstens zwei Jahren versuchen, die 100.000er Marke an Mitgliedern zu überschreiten ${ }^{11}$.

Das im Rundfunkgesetz festgelegte Verhältnis der in den Kategorien A, B und C den Rundfunkgesellschaften zugewiesenen Sendezeit läßt sich in der Formel $5: 3: 1$ ausdrücken, wobei eine Rundfunkgesellschaft der Kategorie $C$ stets mindestens $2^{1 / 2}$ Stunden Sendezeit pro Woche zugewiesen erhält. Durch die Zunahme der Anzahl der Sendeberechtigten, die Beförderung von Rundfunkgesellschaften in eine höhere Kategorie und das relative Zurückbleiben der Sendezeit gegenüber dem gestiegenen Bedarf, kann der im Rundfunkgesetz festgelegte Verteilerschlüssel bereits seit einigen Jahren nicht mehr angewendet werden. Die TROS trat bereits nach einem Jahr (vom 1. Oktober 1967 an) in den C-Status, erwarb infolge eines spektakulären Anwachsens den B-Status (vom 1. Oktober 1971 an) und schließlich den A-Status (vom 1. Oktober 1974 an). Die EO bekam am 1. Oktober 1972 den C-Status.

Das Interesse, die Zielsetzungen neuer Bewerber für eine Sendeerlaubnis zu verwirklichen, muß ausgewogen behandelt werden, weil sonst die Gefahr einer Zersplitterung der verfügbaren Sendezeit besteht. Im Hinblick auf die steigende Anzahl Sendeberechtigter und Gruppen, die ebenfalls Sendezeit verlangen, wurde die Marke von mindestens 15.000 Mitgliedern am 1. Januar 1974 auf 40.000 erhöht. Ubrigens versuchten seit 196620 Gesellschaften, Stiftungen und vergleichbare Gruppen vergebens, Rundfunkgesellschaften mit Bewerber-Status zu werden. Sowohl Rundfunkgesellschaften mit Bewerberstatus als auch die Rundfunkgesellschaften der Kategorien $\mathrm{C}, \mathrm{B}$ und $\mathrm{A}$ müssen ein vollständiges Programm senden. Dieses Programm hat nach der unklaren und jetzt zur Diskussion gestellten Umschreibung des Rundfunkgesetzes in vernünftigem wechselseitigem Verhältnis zumindest Teilbereiche kultureller, informativer, erzieherischer und unterhaltender Art zu umfassen ${ }^{12}$.

Die Offenheit des Systems kommt auch zum Ausdruck in der möglichen Zuweisung von Sendezeiten an Kirchengemeinschaften, Gemeinschaften auf geistlicher Grundlage (Humanistisch Verbond [Humanistischer Verband] und Vrije Gedachte [Freier Gedanke]), politische Parteien (Hörfunk seit 1959 und Sehfunk seit 1962), und - in bestimmten Fällen - andere Einrichtungen, die keine vollständige Rundfunkaufgabe 
anstreben (seit dem 1. Oktober 1967) ${ }^{13}$. Unter die letzte Kategorie der sogenannten „mini-omroepen“ (Mini-Rundfunkgesellschaften) fallen die Radio-Volksuniversiteit (Rundfunk-Volksuniversität), die Stichting Socutera (Stiftung Socutera) und die Onafhankelijke Radio en Televisie Stichting Symbiose (Unabhängige Rundfunkund Fernsehstiftung Symbiose). In der ministeriellen Politik kann man in den letzten Jahren das Streben nach einer Begrenzung der Anzahl der Mini-Rundfunkgesellschaften feststellen. Das Fernsehprogramm "Zienswijze (Sehweise) von der Nederlandse Omroep Stichting (NOS = Niederländische Rundfunkstiftung) - darüber unten mehr - ist zum Beispiel darauf ausgerichtet, alle möglichen Gruppierungen innerhalb des Rahmens der durch die NOS zu verwirklichenden Begegnungsprogramme zu Wort kommen zu lassen, ohne daß diese Gruppierungen eigene Sendezeiten erhalten ${ }^{14}$.

\section{Das Prinzip der Zusammenarbeit im Rundfunk seit 1969}

Die Zusammenarbeit im niederländischen Rundfunk hatte vor 1969 einen freiwilligen, konföderativen Charakter. Das Rundfunkgesetz brachte eine verpflichtende Zusammenarbeit nichtföderativer Art mit sich. Die Nederlandse Omroep Stichting (NOS) ist das gesetzliche Organ für Zusammenarbeit mit einem selbständigen Vorstand, einem Verwaltungsrat und einigen anderen Gremien. Die NRU und die NTS wurden am selben Tag aufgehoben, als der Vorstand der NOS gebildet wurde, nämlich am 29. Mai 1969. Daß die NOS ein Organ für Zusammenarbeit ist, zeigt sich deutlich aus der Zusammensetzung des Vorstands. Von den 28 Mitgliedern vertreten 14 die sieben Rundfunkgesellschaften. Sieben Mitglieder werden von kulturellen und gesellschaftlichen Organisationen entsandt. Die Krone ernennt sieben unabhängige, sogenannte Kron-Mitglieder. Die Geschäftsführung der NOS liegt, nach den Bestimmungen des Rundfunkgesetzes, in den Händen eines Verwaltungsrats. Der Vorstandsvorsitzende, ebenfalls von der Krone ernannt, ist zugleich Vorsitzender des Verwaltungsrats $^{15}$. Zudem sind sechs Vorstandsmitglieder Verwaltungsratsmitglieder.

Die Regierung wollte mit dem Rundfunkgesetz nicht nur - auf der Linie der Tradition - die Verteilung der knappen Sendemöglichkeiten regeln. Sie wollte überdies zum Ausdruck bringen, daß der Staat die Aufgabe hat, auch im Rundfunk das Prinzip der freien Meinungsäußerung und die Informationsfreiheit zu verbürgen und daß der Rundfunk dabei Kultur und Wissen verbreiten und Möglichkeiten zur Unterhaltung anbieten muß. Im Hinblick hierauf wurden auch Kron-Mitglieder und durch die nationalen Kulturorganisationen gewählte Mitglieder in den Vorstand der NOS berufen. Die NOS erhielt mit Bewahrung des pluralistischen Charakters des Rundfunksystems neben der Sorge für die technischen und administrativen Belange auch die Aufgabe, in der ihr zugewiesenen Sendezeit gemeinsame Programme auszusenden ${ }^{16}$. Dieser Aspekt der verpflichtenden Zusammenarbeit führt in zunehmendem Maße zur Reibung mit den Rundfunkgesellschaften, die der NOS Konkurrenz aus einer privilegierten Position heraus vorwerfen. Sie sähen denn auch nicht gern, daß die NOS nach der täglichen ersten abendlichen Nachrichtensendung des Sehfunks ein Magazin mit Nachrichtenanalyse und Hintergrundinformation brächte. Dennoch könnte das in fernerer Zukunft durchaus einmal so werden.

Die NOS ist gesetzlich verpflichtet, sogenannte gemeinsame Hör- und Sehfunkprogramme zu produzieren und zu senden. Diese Programme sollen den Charakter von Begegnungsprogrammen haben, und zwar für die geistigen und gesellschaftlichen 
T'endenzen in der Geselschaft, auch für jene, die nicht durch einen Sendeberechtigten vertreten sind. Ferner geht es bei gemeinsamen Programmen um Sendungen, die nach den Worten des Rundfunkgesetzes - besonders gut für eine gemeinsame Vorbereitung, Erstellung und Ausführung durch die Rundfunkgesellschaften geeignet sind, um ein ausgeglichenes Gesamtbild aller Programme gemeinsam zu verwirklichen. Die Sendezeit der NOS besteht aus mindestens 15 Prozent der gesamten Hörfunksendezeit und zumindest 25 Prozent der gesamten Sehfunksendezeit, für beide mit einem Maximum von 40 Prozent; diese Sendezeit liegt also fest und ist innerhalb der gegebenen Grenzen unantastbar. Die Sendezeit für die Kirchengemeinschaften, Gemeinschaften auf geistiger Grundlage, politische Parteien und die sogenannten „mini-omroepen" darf maximal 10 Prozent der insgesamt verfügbaren Sehfunksendezeit und maximal 5 Prozent der insgesamt verfügbaren Hörfunksendezeit betragen.

Angesichts des spezifischen Charakters der Programme der NOS wurden kraft Rundfunkgesetz zwei Programmräte, einer für Hörfunk und einer für Sehfunk, ins Leben gerufen. Ein Programmrat gibt Richtlinien und Anweisungen an die Mitglieder des Verwaltungsrats der NOS, die mit der Wahrnehmung der Programmangelegenheiten beauftragt $\operatorname{sind}^{17}$. Der Vorsitzende des Programmrats wird von der Krone ernannt, ein Drittel der Mitglieder vom Minister für Kultur, Freizeitgestaltung und Sozialordnung, ein Drittel von den Rundfunkgesellschaften und ein Drittel von den nationalen Kulturorganisationen. Die Kirchengemeinschaften und Gemeinschaften auf geistiger Grundlage können in beide Programmräte beratende Mitglieder ernennen. Jeder Programmrat zählt 22 Mitglieder, abgesehen von den Mitgliedern mit nur beratender Stimme.

Das Prinzip der gemischten Finanzierung des Rundfunks (aus den Erträgen der Rundfunkwerbung und den Rundfunkgebühren einerseits und den Mitgliederbeiträgen der Rundfunkgesellschaften andererseits) kann als ein drittes Merkmal des derzeitigen Rundfunkgesetzes bezeichnet werden. Anfänglich betrug der Mindesbeitrag nur fünf Gulden; dieser Betrag ist am 1. Jan. 1974 verdoppelt worden. Auch können Rundfunkgesellschaften eigenes Kapital, gebildet etwa aus dem Ertrag des Rundfunkprogrammblattes für Programmzwecke einbringen. Die Position des Programmblatts ist übrigens besonders umstritten. Wer es abonniert, wird seit 1969 automatisch Mitglied der Rundfunkgesellschaft, die das betreffende Blatt vertreibt, es sei denn, daß man ausdrücklich wünscht, nur das Programmblatt zu erhalten. Das billigste und kleinste Programmblatt war bis zum vorigen Jahr, als mehr Programmzeitschriften in Miniausgabe auf den Markt kamen, der TROS-Kompaß; sehr viele, aber höchstens 45 Prozent aller Abonnenten einer solchen Zeitschrift lesen sie. Die in der Zukunft wohl zu erwartende Trennung von Abonnement und Mitgliedschaft wird eine Ainderung des Rundfunkgesetzes notwendig machen.

Was die Rundfunkwerbung angeht, so ist zu vermerken, daß 1965 der Werberat und eine Stiftung zum Vertrieb der Werbesendezeit in Hör- und Sehfunk, der heutige STER, gegründet wurden. Der Werberat ist beauftragt, die Tarife festzusetzen und die Normen für Rundfunkwerbung zu formulieren sowie deren Einhaltung zu überwachen. Am 2. Januar 1967 erschien die erste Werbe-Kurzeinblendung auf dem Bildschirm, und am 1. März 1968 wurde mit den Hörfunkwerbesendungen begonnen.

Pro Tag werden zwei Stunden lang für die nördlichen und östlichen Provinzen und während einer Stunde für die südlichen Provinzen regionale Rundfunkprogramme ausgesendet. Zwei seit Ende des Zweiten Weltkriegs bestehende Gesellschaften arbeiten auf dem Gebiet des regionalen Rundfunks unter Verantwortung der NOS ${ }^{18}$. 


\section{Einfluß des „Entsäulungs" prozesses auf das Rundfunksystem und die $K R O$}

$\mathrm{Daß}$ Änderungen in dem übrigens einzigartigen Rundfunkgesetz in der Zukunft nicht ausbleiben können, hat seine Ursachen in Entwicklungen innerhalb des Rundfunksystems selbst wie in der Gesellschaft ${ }^{19}$. Die TROS ist zum Beispiel ein Exponent einer primär kulturell unterhaltenden Rundfunkgesellschaft, die vor 1965 in der „versäulten“ niederländischen Gesellschaft noch undenkbar gewesen wäre. Die Rundfunkgesellschaften, die im Dienst einer bestimmten Parteipolitik und/oder religiöser Überzeugungen standen, veränderten sich ebenfalls. Der „Entsäulungs“prozeß der letzten zehn Jahre ließ diese Rundfunkgesellschaften - ebensowenig wie die damit verwandten oder damit verbundenen gesellschaftlichen Einrichtungen und Organisationen - nicht unbetroffen. Das im Rundfunkgesetz verankerte Mitglieder-Zahlenkriterium wird allzu leicht als einzige oder wichtigste Ursache des Feilschens um die Gunst des Publikums durch die Schaffung mehr unterhaltender Programme angesehen. Dabei wird es so hingestellt, als hätten sie nur ein Auge für die sogenannten Sehdichte- und Bewertungsziffern, die in großer Regelmäßigkeit durch die Abteilung Seh- und Höreruntersuchung der NRU/NTS und ab 1969 durch die NOS erhoben und veröffentlicht werden ${ }^{20}$. Das spektakulärste Wachstum einer Rundfunkgesellschaft war nicht so sehr einer erfolgreichen Publikumswerbung zu danken als vielmehr einer aufsehenerregenden Fusion. Die AVRO ging nämlich 1967 eine Fusion ein mit der Stichting Radio en Televisie Nederland (RTN = Stiftung Rundfunk und Fernsehen der Niederlande), die nicht über eine Sendeerlaubnis, wohl aber über die vielgelesene Programm- und Familienzeitschrift „Televizier" verfügte. Als die AVRO unter anderem durch diesen Schritt die Grenze von 800.000 Mitgliedern überschritt, beanspruchte sie den nicht ausdrücklich im Rundfunkgesetz genannten Status einer Superrundfunkgesellschaft. Das lieferte nicht das begehrte Mehr an Sehfunksendezeit, wohl aber seit dem 1. Oktober 1972 für eine Zeitlang mehr Hörfunksendezeit. Für eine Ubersicht der Sendezeitverteilung auf die Sendeberechtigten zum 1. Januar 1975 verweisen wir auf Tabelle $I I$.

Tabelle II: Die niederländischen Sendeberechtigten und ihre Sendezeit pro Woche für Hörund Sehfunk am 1. Januar 1975.

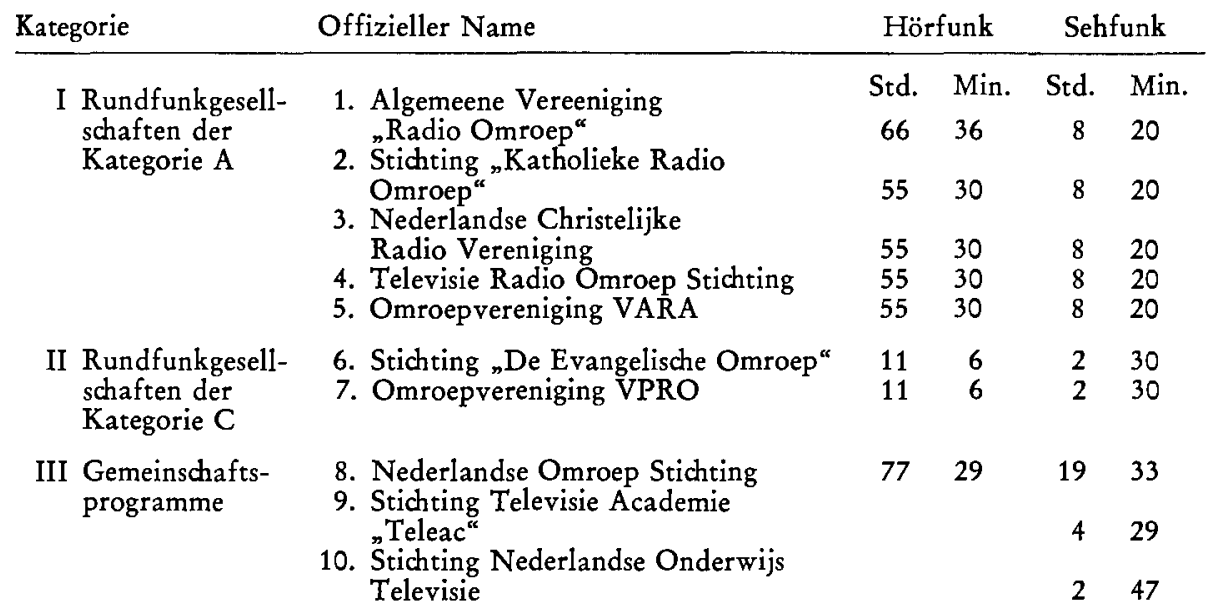


IV Kirchengemein- 11. Interkerkelijk Overleg in schaften

Radio-aangelegenheden

Std. Min. Std. Min.

namens: Nederlandse Hervormde Kerk

Algemene Doopsgezinde Sociëteit

Evangelische Lutherse Kerk

Remonstrantse Broederschap

Oud-Katholieke Kerk van

Nederland

Evangelische Broedergemeenten

12. Convent van Kerken inzake

Radiodiensten

namens: Gereformeerde Kerken in Nederland idem, vertegenwoordigd in de

Generale synode van Enschede 1955

Christelijke Gereformeerde Kerken

Bond van Vrije Evangelische

Gemeenten

Unie van Baptisten Gemeenten

Leger des Heils

13. Rooms Katholiek Kerkgenootschap*

14. Kerkgenootschap der

Zevende-Dag Adventisten

15. Christian Science Committee on publication for the Netherlands

$\mathrm{V}$ Vereinigungen

16. Humanistisch Verbond

für Geistesleben

17. Vereniging "De Vrije Gedachte"

VI Politische

18. Katholieke Volkspartij

Parteien

19. Partij van de Arbeid

20. Volkspartij voor

Vrijheid en Democratie

21. Anti-Revolutionaire Partij

22. Christelijk Historisdhe Unie

23. Communistische Partij Nederland

24. Democraten '66

25. Politieke Partij Radicalen

26. Democratisch Socialisten ' 70

27. Boerenpartij

28. Pacifistisch Socialistische Partij

29. Gereformeerd Politiek Verbond

30. Rooms-Katholieke Partij Nederland

VII Übrige

Einrichtungen

31. Stichting Radio Volksuniversiteit

32. Stichting "Bond zonder Naam"

33. Stichting Socutera

34. Onafhankelijke Radio en Televisie Stichting "Symbiose"

Zahlen für 11-15 insgesamt:

$\begin{array}{llll}7 & 37^{1} / 2 & 2 & 351 / 2\end{array}$

Zahlen für 16-17 insgesamt: 34 9

\section{Spezielle Sendungen u. a. Sendungen $* *$}

35. Sendungen der Regierung („Regeringsuitzendingen“)

36. Werbesendungen (Stichting Etherreclame)

(Stichting Etherreclame)

Insgesamt pro Woche

* RKK hat die KRO mit der Realisierung der Sendungen beauftragt.

$*$ Nicht genannt sind der Regionalhörfunk, die Nachrichtensendungen der Presseagentur "Algemeen Nederlands Persbureau“, der Schulfunk („Stichting Nederlandse Schoolradio") usw.

Quelle: Mitteilungen der „Nederlandse Omroep Stichting“. 
Die sechziger Jahre haben die KRO ebenso wie die anderen profilierten weltanschaulichen Rundfunkgesellschaften VARA, VPRO und NCRV neben dem hier oben behandelten Konkurrenzproblem mit der Frage nach dem Selbstverständnis konfrontiert. Fast vierzig Jahre lang hatte die KRO eine sehr klare Funktion innerhalb der katholischen Gemeinschaft inne. Sie war primär eine Rundfunkgesellschaft von Katholiken für Katholiken und ihre Organisationen. Im „Entsäulungs“prozeß der sechziger Jahre wurde die KRO ein Rundfunk von vornehmlich Katholiken für jeden Niederländer und mehr im besonderen für niederländische Katholiken. Im Jahre 1963 wurde die Zielsetzung der KRO in Artikel 2 der Statuten neu formuliert; sie lautet seither: "Die Stiftung hat zum Ziel, Hör- und Sehfunk zur geistigen Entfaltung, Unterrichtung und Unterhaltung der Hörer und Seher einzusetzen. Um ihre Aufgabe zu erfüllen, anerkennt sie Gottes Offenbarung und bekennt sie so, wie diese durch die katholische Kirche bewahrt und weitergegeben wird." Die führende Aufgabe wurde umgebildet zu einer mehr begleitenden. Ein 1962 errichteter Programmberatungsausschuß, bestehend aus Fachleuten in den Bereichen Religion, Politik und Kultur, bekam zur Aufgabe, die Hör- und Sehfunkprogramme kritisch zu verfolgen, die Programmpolitik zu beraten und Vorschläge für die zu erstellenden Programme zu machen. In den Jahren 1969 und 1970 haben die Mitarbeiter aller Abteilungen innerhalb der KRO ihr katholisches Selbstverständnis überdacht. Sie kamen zu dem Schluß, daß die Verbundenheit mit den Katholiken als Bevölkerungsgruppe bei der Verwirklichung der Zielsetzungen der KRO noch immer bedeutsam sei. Die KRO solle überdies ein Resonanzboden sein und einen Dialog zu ermöglichen suchen zwischen den unterschiedlichen Standpunkten, insbesondere innerhalb der katholischen Kreise. Diese Pluriformität solle jedoch nicht zum Verzicht auf eine eigene Stellungnahme führen dürfen. Praktisch-programmatisch solle das heißen, daß zur Standortbestimmung und Meinungsbildung des Sehers und/oder des Hörers beigetragen werden könne, insofern man Tatsachen nicht nur registriere und signalisiere, sondern auch durchleuchte und kommentiere. Die Aufmerksamkeit für jene, die in der Gesellschaft an den Rand gedrängt wurden oder sogar an ihr zerbrachen, wird in den letzten fünf Jahren deutlicher in den Programmen zum Ausdruck gebracht ${ }^{21}$. Der heutige Minister Van Doorn (für Kultur, Freizeitgestaltung und Sozialordnung) war von 1961 bis 1973 in einer äußerst wichtigen und mühseligen Zeit der erste Laienvorsitzende der $\mathrm{KRO}^{22}$. In einem Interview mit G. Puchinger über das Thema Polarisierung behauptete Van Doorn 1972, die KRO habe in den letzten zehn Jahren in recht abgewogener Weise Farbe bekannt. Das bedeutete eine Entscheidung für die Erneuerung und die nachkonziliare Linie, wobei jedoch auch andere legitime Auffassungen innerhalb der untereinander geteilten katholischen Gemeinschaft der Niederlande zu Worte kommen sollten ${ }^{23}$. Die zunehmende Polarisierung unter den Katholiken führte zur Kritik an der KRO vor allem durch die Konservativen, die meinten, daß die katholische Rundfunkgesellschaft nicht mehr ihrer Zielsetzung entspreche. Uber die Jahre 1972 und 1973 verlor die KRO zunächst mehr Mitglieder, als neue hinzukamen. Am 1. Januar 1975 hatte die KRO jedoch wieder $11.868 \mathrm{Mit}-$ glieder mehr als ein Jahr zuvor, während die protestantisch-christliche NCRV im gleichen Jahr ein gleiches Wachstum erlebte. Uberdies war inzwischen klar geworden, daß der 1971 gegründete Rooms Katholieke Zender der Stichting Sint Willibrord in seinem Streben nach einer konservativen katholischen Rundfunkgesellschaft scheitern sollte. Anfang 1975 soll die Anzahl der Mitglieder nur 8.000 betragen haben, was noch weit von der gesetzlich geforderten Grenze von 40.000 entfernt liegt. 
Bernhard Kardinal Alfrink bestätigte während eines Gedächtnistreffens Ende 1974 aus Anlaß des vierzigjährigen Bestehens der Hörfunk-Aktualitäten-Rubrik „Echo" (Nachrichtenmagazin) der KRO, daß das Gesamtbild einer Rundfunkgesellschaft nicht auf eine bestimmte Art von Programmen gegründet sein dürfe ${ }^{24}$. Gerade zuvor hatte der bekannte katholische Politiker Prof. P. Steenkamp sich als Mitglied der KRO streichen lassen, weil die Rubrik „Echo“ einseitig links sei. Der konservative Bischof von Roermond, Msgr. J. M. Gijsen, war bereits früher abgeschwenkt. Anfang Februar 1975 äußerte sich der Episkopat nichtsdestoweniger positiv über die KRO; vornehmlich fand - so ein Pressebericht - der Ernst, den die KRO mit dem katholischen Selbstverständnis mache, Zustimmung.

\section{Die Zukunft für einen katholischen Rundfunk und Schlußfolgerung}

Als Ansatz für ein auf die Zukunft gerichtetes Funktionieren der KRO ist 1973 eine neue Vorstandsstruktur zustande gekommen. Sie war 47 Jahre lang unverändert geblieben. Nur der 1962 eingerichtete Programmberaterausschuß kann als ein neues Element vermerkt werden. Der geistliche Berater war 1939 zum Bischöflichen Kommissar ernannt worden, aber als Mitglied des Vorstandes (ohne Stimmrecht) blieb er bis heute das einigende Band zwischen Episkopat und KRO.

Der Kontakt mit den Mitgliedern der KRO - bei einer Stiftung sowieso schwach lief anfänglich über Delegierte des Nederlandse Bond van R. K. Radiovereenigingen, die vor allem als Mitglieder-Werbezentren funktionierten. Auf Wunsch des Episkopats wurde diese Organisation 1931 durch fünf autonome Diocesane Bonden (Diözesane Verbände) ersetzt, die in einer Föderation zusammenarbeiteten. Diese Struktur erwies sich als erfolglos und wurde durch fünf und später sieben Diocesane Contact Commissies (Diözesane Kontaktkommissionen) ersetzt, deren Mitglieder durch örtliche Aktionsausschüsse entsandt wurden.

Recht spät, nämlich am 31. August 1973, trat ein Vorstand neuen Stils für die KRO erstmalig zusammen. Der Gesamtvorstand besteht nunmehr aus dem Vorsitzenden, dem Bischöflichen Kommissar, 15 Abgeordneten der großen katholischen Organisationen und 14 Abgeordneten der Diözesanen Kontaktkommissionen, aber neben ihnen fanden 14 Mitglieder Platz, die aus den Mitarbeitern der KRO gewählt sind. Die 45 Mitglieder des neuen Gesamtvorstandes wählten sechs des aus sieben Mitgliedern bestehenden Vorstandsausschusses, wobei jeweils zwei aus den Bereichen Organisationen, Mitarbeiter und Mitglieder kommen mußten ${ }^{25}$. Das siebte Mitglied des Vorstandsausschusses ist der Vorsitzende.

Mit diesem ernsthaften Demokratisierungsbemühen, das in der neuen Vorstandsstruktur zum Ausdruck kommt, ist die Frage des Selbstverständnisses natürlich noch nicht gelöst. Sechs Diskussionsberichte zirkulieren im fünfzigsten Jahr, in dem die KRO nunmehr besteht, auf verschiedenen Ebenen der Vorstandsstruktur; sie sollen die Grundlage bilden für eine Zielsetzung, in der das " $\mathrm{K}$ “ der KRO eine wichtige Rolle spielen wird. Seit dem Erfolg der konservativen EO rechnet die NCRV stärker mit den unterschiedlichen Tendenzen innerhalb des niederländischen Protestantismus; sie befindet sich in einer mit der KRO vergleichbaren Position. Es ist unseres Erachtens schade, daß noch keine Rede ist von Zusammenarbeit mit dem Blick auf eine Fusion zwischen KRO und NCRV. Die VARA droht bisweilen der Last interner ideologischer und anderer auf personaler Ebene liegenden Auseinandersetzungen zu erliegen. Die VPRO entwickelte sich seit der Änderung der Statuten 1968 von einer 
freigeistig protestantischen Rundfunkgesellschaft $\mathrm{zu}$ einem links progressiven, fast avantgardistischen $\mathrm{Kreis}$ von gut 100.000 Intellektuellen.

In den letzten Jahren wurde seitens progressiver Politiker und Journalisten für ein System von Rundfunkwahlen als Basis für Sendezeitverteilung plädiert. Am konkretesten ausgearbeitet ist der Vorschlag einer Kommission unter Vorsitz von J. Nagel von der sozialistischen Partij van de Arbeid (Arbeitspartei) aus dem Jahre 1972: Die Rundfunkwahlen sollten alle vier Jahre einmal abgehalten werden; Rundfunkgesellschaften, die mehr als 12 Prozent der gültigen Stimmen erhielten, sollten vier Jahre lang den A-Status bekommen, bei einem Ergebnis von zwischen 8 und 12 Prozent der Stimmen sollte der B-Status und bei einem Ergebnis von zwischen 4 und 8 Prozent der Stimmen sollte der C-Status zugesprochen werden müssen. Eine Probeuntersuchung brachte 1974 interessante Ergebnisse. Wir geben sie in Tabelle III wieder, auch wenn Minister Van Doorn - mit guten Gründen, so meinen wir - in seinem Memorandum über Medienpolitik Rundfunkwahlen nicht als Lösung der Probleme um das heutige System der Sendezeitzuteilung ansieht ${ }^{20}$.

Tabelle III

\begin{tabular}{lccc}
\hline & $\begin{array}{c}\text { Prozentsatz } \\
\text { nach Probewahlen } \\
1974^{27}\end{array}$ & $\begin{array}{c}\text { Prozentsatz } \\
\text { nach Anzahl der } \\
\text { Mitglieder } \\
\text { (heutiges System) }\end{array}$ & $\begin{array}{c}\text { Prozentsatz } \\
\text { nach Probeuntersuchung } \\
\text { NOS (1972; bei Perso- } \\
\text { nen von 20 Jahren } \\
\text { und älter) }\end{array}$ \\
\hline TROS & 25,3 & 15,8 & 22 \\
VARA & 18,1 & 15,7 & 11 \\
AVRO & 14,8 & 26,9 & 15 \\
NCRV & 14,8 & 15,3 & 11 \\
KRO & 12,6 & 17,0 & 11 \\
VPRO & 11,2 & 4,5 & 3 \\
EO & 3,3 & 4,8 & 2
\end{tabular}

Daß die TROS nach diesen Ergebnissen im Fall von Rundfunkwahlen einer rosigen Zukunft entgegenginge, braucht nach dem, was wir oben schrieben, kein Staunen zu erregen, überraschend ist vielmehr ihr Vorsprung gegenüber ihrem jetzt noch starken Konkurrenten AVRO.

Anders als sich aus diesen globalen Untersuchungen erweisen kann, meinen wir bei den noch relativ günstigen Ergebnissen für die KRO doch ein Fragezeichen setzen zu müssen. Die Zukunft der KRO hängt jedoch von vielen Faktoren ab: der künftigen Entwicklung unter den Katholiken in den Niederlanden; den zu erwartenden Änderungen im Rundfunkgesetz; dem Ergebnis des regionalen und lokalen Rundfunks neuen Stils und den davon vielleicht zu erwartenden Änderungen im Verhalten der Sehfunkzuschauer; ferner von der Entwicklung des erziehenden und unterweisenden Sehfunks, der Position der NOS als Sendeberechtigter, dem Erfolg oder Scheitern der Regierungspolitik, die sich auf das Eindämmen der Unterhaltungsfunktion des Sehfunks auf Kosten aller anderen Funktionen in einer demokratischen Gesellschaft richten müßte. Ferner hängt sie $a b$ von der Frage, wie lange innerhalb der KRO Mitarbeiter gefunden werden, die bereit sind, aus einer Position der Mitte ihre Aufgabe zu erfüllen - die dabei von Zeit zu Zeit einen Eiertanz aufführen müßten, um die durch viele Gruppen listig in das KRO-Nest gelegten Eier nicht zu zerbrechen. Die katholische Tageszeitung „De Tijd“ ist - trotz Interesses an ihrer 
Dialog- und Forum-Funktion - inmitten polarisierender und disputierender Glaubensgenossen untergegangen. Der KRO sei im Jahr des goldenen Jubiläums eine bessere Zukunft vergönnt, auch im Interesse des gesamten niederländischen Rundfunksystems.

\section{Anmerkungen:}

1. Die hier gebotene knappe Ubersicht über die Entwicklung des niederländischen Rundfunks ist eine Zusammenfassung verschiedener niederländischsprachiger Publikationen des Verfassers dieses Artikels. Vgl. die neueste Ubersicht: J. Hemels: Omroep, in: P. van Dijke, J. M. Galjaard, J. M. H. J. Hemels, W. H. Kuipers en A. J. van't Veer (Hrsg.): Handboek besturen in openheid, Alphen/Rijn 1975, S. VII-3, 1-26. Siehe auch: H. Schaafsma: Geschiedenis en karakteristiek van de omroep, Amsterdam 1970; H. van Pelt: De omroep in revisie. Ontwikkeling van het radio- en televisiebestel in Nederland en Belgie. Een vergelijkende studie, Löwen 1973; Anthony Smith: The Dutch system: The pillars of Hilversum, the issue of access, in: The shadow in the cave. A study of the relationship between the broadcaster, his audience and the state, London 1973, S. 264-278; W. B. Emery: National and international systems of broadcasting. Their history, operation and control, East Lansing 1969, S. 140-157 ("The Netherlands: pluralism with freedom"), und B. Schmitz: Die Neuordnung des Rundfunkwesens in den Niederlanden. Sonderdrudk aus: Jahrbuch für Internationales Recht, 13. Bd., Göttingen 1967, S. 338-371.

2. Vgl. A. Lijphart: The politics of accomodation. Pluralism and democracy in the Netherlands, Berkeley - Los Angeles 1968, en J. M. G. Thurlings: De wankele zuil. Nederlandse katholieken tussen assimilatie en pluralism, Nijmegen - Amersfoort 1971.

3. H. Algra, C. Rijnsdorp en B. van Kaam: Vrij en gebonden. 50 jaar NCRV, Baarn 1974.

4. J. Bank: Een halve eeuw omroephistorie in hoofdlijnen, in: Historie en perspektieven (gedenkboek van de AVRO), Hilversum 1973, S. 9-48.

5. Das Rundfunkgesetz von 1928 war tatsächlich kein eigenständiges Gesetz, sondern beinhaltete eine Anderung des Telegraphen- und Telephongesetzes von 1904. Alle gesetzlichen Regelungen in Hinsicht auf Hör- und Sehfunk stützten sich bis 1969, als das Rundfunkgesetz in Kraft trat, auf dieses Gesetz aus dem Jahre 1904.

6. Vgl. Gabriele Hoffman: NS-Propaganda in den Niederlanden. Organisation und Lenkung der Publizistik unter deutscher Besatzung 1940-1945, München/Pullach - Berlin 1972, S. 230-241, und Dick Verkijk: Radio Hilversum 1940-1945. De omroep in de oorlog, Amsterdam 1974.

7. Wir haben für die Ubersicht über die Geschichte der KRO nichtpublizierte Notizen des Archivars der KRO, Heinz Joosten, verwenden dürfen, dem wir dafür sehr dankbar sind.

7a. Vgl. Charles E. Sherman: The International Broadcasting Union. A study in practical internationalism, in: „EBU Review“, Bd. 25, 1975 Nr. 3, S. 32-36.

8. Siehe für den REM-Versuch: Karel Remes: Prevention of broadcasts transmitted on installations on the high seas. Dutch legislation on installations on the high seas, in: „EBU Review“, Nr. 90b (März 1965), S. 47-52, und Albert Namurois: The prevention of the activities of ${ }^{n}$ pirate ${ }^{*}$ broadcasting stations. A European agreement, Straßburg Dezember 1964, in: ibidem, S. 36-46.

9. Eine deutsche Übersetzung des Rundfunkgesetzes ist nicht vorhanden, wohl eine englische: Broadcasting Act, Hilversum (NOS), o. J., und: Broadcasting Decree of 1st April 1969, containing regulations for the execution of the provisions of the Broadcasting Act, Hilversum (NOS), o. J. Die beste, mit Anmerkungen versehene niederländische Ausgabe des Rundfunkgesetzes findet sich in: Omroepwet van 1 maart 1967, Staatsblad nr. 176, tot regeling van de omroep en wet op de omroepbijdragen van 20 december 1968 , Staatsblad 687, tot regeling van de omroepbijdragen, bewerkt door M. P. Bajetto, Chef etc., Zwolle 1969 (Ausgabe Schuurman \& Jordens, Nr. 164).

10. Vgl. Art. 13 Rundfunkgesetz. 
11. Vgl. Art. 14 Rundfunkgesetz.

12. Vgl. Art. 35 Rundfunkgesetz.

13. Vgl. Art. 16 bis 19 Rundfunkgesetz.

14. Vgl. Art. 36 Rundfunkgesetz.

15. Vgl. Art. 43 Rundfunkgesetz.

16. Vgl. Art. 36 Rundfunkgesetz.

17. Vgl. Art. 44 Rundfunkgesetz.

18. Vgl. Art. 47 Rundfunkgesetz.

19. Siehe J. Hemels: Onweerswolken boven Hilversum? Een kritische beschouwing van het Nederlandse omroepbestel, in: Media, 88:1973/74, S. 9-17.

20. Die Ziffer der Zuschauerdichte für ein Programm setzt sich aus dem Prozentsatz der zwölf Jahre alten und älteren Bevölkerung zusammen, die ein Programm wenigstens zur Hälfte gesehen hat. Angesichts der Tatsache, daß praktisch 100 Prozent der zehn Millionen zwölfjährigen und älteren Niederländer zuhause eine Fernsehmöglichkeit haben, steht 1 Prozent Zuschauerdichte für 100.000 Personen.

Die Bewertungsziffer für ein Programm ist der Schnitt der Bewertungsziffern, die die Teilnehmer in dem Continu Programma Onderzoek der NOS diesem Programm zuerkennen. Die Bewertungsziffern gehen von 1 bis 10 , wobei der Schnitt aus praktischen Gründen ohne Komma vorgelegt wird. Am 1. Januar 1965 hat man in den Niederlanden mit der ständigen Programmuntersuchung begonnen.

21. Jac. Grijpink: KRO: aanpassen aan veranderende tijden, in: „Kruispunt“, 7:1971, Nr. 2, S. 8-11. Vgl. auch: Massamedia en levensbeschouwing. „KRO-Cahier“ 1971-1, Hilversum 1971.

22. Die Vorsitzenden aus dem Orden der Dominikaner waren nach Perquin hintereinander: J. Dito (1938-1945), J. Kors (1945-1959) und E. van Waesberge (1959-1961). Van Doorn war jahrelang Mitglied der Zweiten Kammer für die Katholieke Volks-Partij; als 1967 die mehr progressive Politieke Partij Radicalen ins politische Leben eintrat, wurde Van Doorn Mitglied dieser neuen Partei. Seit Van Doorn Minister ist (1973), ist Th. Loerakker Vorsitzender.

23. Zitiert in: Okke Jager: Baas boven buis. De televisie in theorie en praktijk, Kampen 1974 , S. $148-149$.

24. Toespraak van Bernardus Kardinal Alfrink bij gelegenheid van het veertigjarig bestaan van de KRO-radio-aktualiteiten, Noordwijkerhout, 18 december 1974; vgl. CS 8:1975, Nr. 3, S. 262.

25. J. Hemels: De KRO-verkiezingen en de machtige organisaties, in: „De Bazuin“, 56:1973, Nr. 20, S. 5-6.

26. Nota over het massamedia-beleid, 's-Gravenhage 1975.

27. Omroepverkiezingen, Amsterdam (Inter/View) 1974.

28. Programmabladen (Rapport nr. 90 van de afdeling Luister- en Kijkonderzoek van de NOS), Hilversum 1973, S. 18. Siehe auch: Omroepverkiezingen in Nederland. Een onderzoek naar de opkomst en uitslag van omroepverkiezingen, uitgevoerd tijdens de gemeenteraadsverkiezingen van 1974, Hilversum (NOS) 1974.

\section{S U M M A R Y}

Catholic broadcasting in the Netherlands, past and present, are discussed on the occasion of the 50th anniversary of the Catholic Broadcasting Organization KRO (Katholieke Radio Omroep). Drawing lines from the development up till now, a view can be attempted towards the future of catholic broadcasting in the Netherlands. The KRO as an institution representing catholic broadcasting for half a century succeeded also in the last decennium 
to maintain its strong position in the national broadcasting system. Changes in Church and society have not ended this situation, as has been with the catholic daily press in this country. But one can ask, if this situation is not going to be changed soon. Through changes in the Dutch Radio system caused by a different mentality of the listeners towards ideological broadcasting societies the specific aim of the KRO and her self-understanding might be under pressure. The growing polarization within the catholic population will lead towards different expectations from the KRO. One must ask if the strategy of the KRO in the 60's to be a forum will not lead towards a limited and finally dying development. Nobody can predict in how far media politics will bring a better climate for the outstanding broadcasting societies. The author does not trust such a politically defined backing and pleas for a stronger KRO in cooperating with the protestant NCRV and maybe later, a fusion of these two.

\section{RÉ SUM É}

L'auteur traite - à l'occasion du 50e anniversaire de la «Stichting Katholieke Radio Omroep» (KRO = Fondation de la Radio Catholique) - du passé et du présent de la radio catholique aux Pays-Bas devant l'arrière-plan du système de radiodiffusion néerlandais dans son ensemble. A partir des lignes de développement qu'il esquisse, il essaie de développer une perspective d'avenir de la radio catholique aux Pays-Bas. La conclusion est que la KRO en tant qu'institut qui défend l'idée de la radio catholique depuis maintenant un demi-siècle a réussi à affirmer, dans la dernière décennie également, une position forte dans le système de radiodiffusion du pays. Des transformations décisives dans l'Eglise et dans la société ne lui ont pas, à l'opposè de la presse journalière aux Pays-Bas, porté un coup fatal.

Il est cependant question de savoir si la sécurité de vie de la KRO n'est pas une sécurité apparente. La fixation du but de la KRO et son autocomprehension propre qui en découle sont livrées à une contrainte constamment forte par suite de décalages dans le système de radiodiffusion néerlandais suivant une transformation du point de vue du public face aux offices idéologiques de radiodiffusion. Le processus progressif de polarisation au sein des catholiques amènera à un modèle différencié d'attente en ce qui concerne la KRO, pour autant que ce groupe de population continue à attacher de la valeur $\grave{a}$ un office catholique de radiodiffusion.

Une question se pose: à savoir si la fonction de forum choisit de façon consciente dans les années 60 ne mène pas la KRO sur une voie étroite et, en fin de compte, dans une impasse. On ne peut pas prévoir à quel point la politique des mass-media saura créer pour les offices de radiodiffusion profilés un climat plus favorable qu'il ne l'est en ce moment. L'auteur n'a cependant pas confiance en une telle couverture de l'arrière, à conviction politique, et plaide pour un renforcement de la KRO par l'intermédiaire d'une collaboration avec la NCRV chretienne protestante et peut-être plus tard d'une fusion avec elle.

\section{R ES U M E N}

Con motivo del 50 aniversario de la "Stichting Katholieke Radio Omroep" (KRO = Fundación Católica de Radiodifusión), el autor se ocupa del pasado y el presente de la radiodifusión católica en Holanda, considerada en le marco global del sistema radiofónico holandés. Fundándose en las líneas de desarrollo que describe, trata de mostrar una perspectiva del futuro de la radiodifusión católica en Holanda. Su conclusión final es que la $\mathrm{KRO}$, como institución que representó durante medio siglo la concepción de una radio católica, logró incluso durante la última década mantener una posición destacada entre las emisoras del país. Las profundas transformaciones en la Iglesia y en la sociedad no significaron para ella un golpe mortal como lo fueron para la prensa católica en Holanda. 
Persiste el interrogante de si estas garantías son sólo una pseudoseguridad para la KRO. Los cambios registrados en el sistema radiofónico holandés, consiguientes a la actitud cambiante del público ante las emisoras de radio ideológicas, constituyen una presión grave y permanente para la meta específica de la KRO y para su propio concepto. Mientras los católicos sigan considerando útil una emisora católica, el proceso de polarización dentro de este grupo de población conducirá a una diferenciación ejemplar y esperanzadora con respecto a la KRO.

También es un problema si la función de foro elegida conscientemente por la KRO durante la década de los años sesenta no conducirá a una vía estrecha y en definitiva a una vía muerta.

No puede predecirse en qué medida una política de medios de communicación social es capaz de crear un clima, más propicio que el existente, para la proliferación de emisoras perfiladas. El autor desconfía de un respaldo determinado, precisamente político, y preconiza el fortalecimiento de lo KRO mediante la cooperación con la emisora protestante NCRV y tal vez la fusión posterior de ambas. 